\title{
Limitation on Prepulse Level for Cone-Guided Fast-Ignition Inertial Confinement Fusion
}

\author{
A. G. MacPhee, ${ }^{1, *}$ L. Divol, ${ }^{1}$ A. J. Kemp, ${ }^{1}$ K. U. Akli, ${ }^{2}$ F. N. Beg, ${ }^{3}$ C. D. Chen, ${ }^{1}$ H. Chen, ${ }^{1}$ D. S. Hey, ${ }^{1}$ R. J. Fedosejevs, \\ R. R. Freeman, ${ }^{5}$ M. Henesian, ${ }^{1}$ M. H. Key, ${ }^{1}$ S. Le Pape, ${ }^{1}$ A. Link, ${ }^{4}$ T. Ma, ${ }^{1,3}$ A. J. Mackinnon, ${ }^{1}$ V. M. Ovchinnikov, ${ }^{5}$ \\ P. K. Patel, ${ }^{1}$ T. W. Phillips, ${ }^{1}$ R. B. Stephens, ${ }^{2}$ M. Tabak,,${ }^{1}$ R. Town, ${ }^{1}$ Y. Y. Tsui, ${ }^{4}$ L. D. Van Woerkom,${ }^{5}$ \\ M. S. Wei, ${ }^{3}$ and S. C. Wilks ${ }^{1}$ \\ ${ }^{1}$ Lawrence Livermore National Laboratory, Livermore, California, USA \\ ${ }^{2}$ General Atomics, San Diego, California, USA \\ ${ }^{3}$ Department of Mechanical and Aerospace Engineering, University of California-San Diego, La Jolla, California, USA \\ ${ }^{4}$ Department of Electrical and Computer Engineering, University of Alberta, Alberta, Canada \\ ${ }^{5}$ College of Mathematical and Physical Sciences, The Ohio State University, Columbus, Ohio, USA
}

(Received 5 October 2009; published 4 February 2010)

\begin{abstract}
The viability of fast-ignition (FI) inertial confinement fusion hinges on the efficient transfer of laser energy to the compressed fuel via multi-MeV electrons. Preformed plasma due to the laser prepulse strongly influences ultraintense laser plasma interactions and hot electron generation in the hollow cone of an FI target. We induced a prepulse and consequent preplasma in copper cone targets and measured the energy deposition zone of the main pulse by imaging the emitted $K_{\alpha}$ radiation. Simulation of the radiation hydrodynamics of the preplasma and particle in cell modeling of the main pulse interaction agree well with the measured deposition zones and provide an insight into the energy deposition mechanism and electron distribution. It was demonstrated that a under these conditions a $100 \mathrm{~mJ}$ prepulse eliminates the forward going component of $\sim 2-4 \mathrm{MeV}$ electrons.
\end{abstract}

Cone-guided fast-ignition inertial confinement fusion (FI) depends on the efficient transfer of laser energy to a forward directed beam of $\sim 2 \mathrm{MeV}$ electrons at the tip of a hollow cone embedded in the side of an inertialconfinement fusion fuel capsule [1]. This scheme is particularly susceptible to laser prepulse [2,3] as the cone wall confines the expanding preformed plasma $[4,5]$ increasing both density scale lengths and laser beam filamentation [6].

The igniter laser pulse requirements for fast ignition depend on the conversion efficiency from laser energy to hot electrons [7], the electron energy spectrum [8], the electron transport efficiency to the ignition hot spot $[9,10]$, and the electron energy deposition efficiency in the hot spot [10]. The required laser energy has been estimated at approximately $100 \mathrm{~kJ}$ in a $20 \mathrm{ps}$ pulse $[1,11]$. Since the ignition hot spot diameter is $\sim 40 \mu \mathrm{m}$, the cone tip must be similar in diameter and the laser intensity $\sim 4 \times 10^{20} \mathrm{~W} / \mathrm{cm}^{2}$. Existing petawatt class laser systems deliver up to $1 \mathrm{~kJ}$ with typical energy contrast $\sim 1 \times 10^{-5}$ and with nonlinear devices this ratio can be improved by a further order of magnitude [12]. Contrast due to amplified superfluorescence and spontaneous emission is independent of the final laser energy; hence, for an ignition pulse of $100 \mathrm{~kJ}$ the prepulse energy on target could range from $100 \mathrm{~mJ}$ to $1 \mathrm{~J}$. Recent work by Baton et al. [5] has shown that some amount of prepulse can strongly affect coupling to cones; however, a detailed understanding of this limit has not been reported.

In this Letter we report recent studies of laser interactions with hollow cone targets comparing simulations and experiments in conditions approaching full fast ignition (FI) using prepulse up to $100 \mathrm{~mJ}$ with main pulse irradiance $\sim 10^{20} \mathrm{~W} \mathrm{~cm}^{-2}$ for picosecond durations. These parameters were accessible using the Titan laser at LLNL, which delivers $(150 \pm 10) \mathrm{J}$ in $(0.7+/-0.2)$ ps at $1 \mu \mathrm{m}$ with $\sim 10 \%$ of the energy deposited above an intensity of $\sim 10^{20} \mathrm{~W} \mathrm{~cm}^{-2}$ at best focus, as described in [13].

We compare coupling for two well-characterized prepulse conditions: (1) an intrinsic Titan laser prepulse with $(7.5 \pm 3) \mathrm{mJ}$ in $1.7 \mathrm{~ns}$ at $7.5 \times 10^{10} \mathrm{~W} \mathrm{~cm}^{-2}$ and (2) $(100 \pm 3) \mathrm{mJ}, 3.0 \mathrm{~ns}$ prepulse at $\sim 10^{12} \mathrm{~W} \mathrm{~cm}^{-2}$. The larger prepulse was generated by injecting an auxiliary laser pulse into the short pulse amplifier chain prior to the main pulse. Targets were $1 \mathrm{~mm}$ long copper cones with $30^{\circ}$ coangle, $25 \mu \mathrm{m}$ wall thickness, and $30 \mu \mathrm{m}$ internal tip diameter. Copper was chosen because copper $K_{\alpha}$ line emission is accessible to proven diagnostics. The energy distribution for the intrinsic prepulse, the auxiliary prepulse and the main pulse were measured by sampling beam leakage through the last turning mirror prior to the final focusing optic, at a plane equivalent to the focal plane on target [13]. Best focus for both the prepulse and the main pulse was set at the inside surface of the cone tip.

The system was modeled in two parts: (i) The radiativehydro code HYDRA [14] was used to calculate the distribution of the preformed plasma created by laser ablation from the inside wall of the cone due to the prepulse; (ii) the plasma simulation code PSC [15] was used to perform a massively parallel particle in cell (PIC) simulation of the ultraintense short pulse laser interaction with the pre- 
formed plasma and to calculate the spatial distribution of hot electrons as a function of time. The two-dimensional PIC simulations of the laser-cone interactions were all performed at full spatial and temporal scales. 20 cells per wavelength and 50 particles per cell were used. The density of the cone wall was clamped at $100 \times$ critical $\left(n_{c}\right)$ to avoid numerical heating. The initial conditions for the PIC simulation were imported from a 2D hydrodynamic simulation performed using HYDRA [14] to model the actual target geometry and the experimentally measured prepulse (both intrinsic and external). The near field intensity distribution of the Titan laser was reconstructed from the measured high power focal spot, resulting in an aberrated spot consistent with experiment. This near field was used as a boundary condition for the Maxwell solver in the PIC simulation. In order to capture the correct preplasma evolution, the PIC simulation was started at $3 \%$ of peak laser power, 1 ps before the peak. Significant channel preformation was observed during the first picosecond, which was able to subsequently guide the laser light near peak power. It should be noted that using a realistic focal spot, preplasma and starting the simulation at very low power lead to significant differences compared to an ideal simulation (Gaussian spot simulated for a FWHM) in terms of channel formation and energy deposition.

In the experiment the time integrated distribution of $K_{\alpha}$ fluorescence radiation from copper cone targets was measured using a spherically bent quartz crystal x-ray microscope tuned to $\mathrm{Cu} K_{\alpha}$ radiation at $8048 \mathrm{eV}$ with $\sim 5.2 \mathrm{eV}$ bandwidth [16-18] and a magnification of $\times 7$ as shown in Fig. 1. Under these conditions the dominant excitation of $K_{\alpha}$ is from binary collisions with the hot electrons; hence, the $\mathrm{Cu} K$-shell emission correlates closely with the distribution of hot electrons $[4,19]$.

As shown in previous work [17] there is direct correspondence between the measured $K_{\alpha}$ at a given point in the image and the calculated hot electron density. The energy lost per $K_{\alpha}$ event is small compared to the average energy

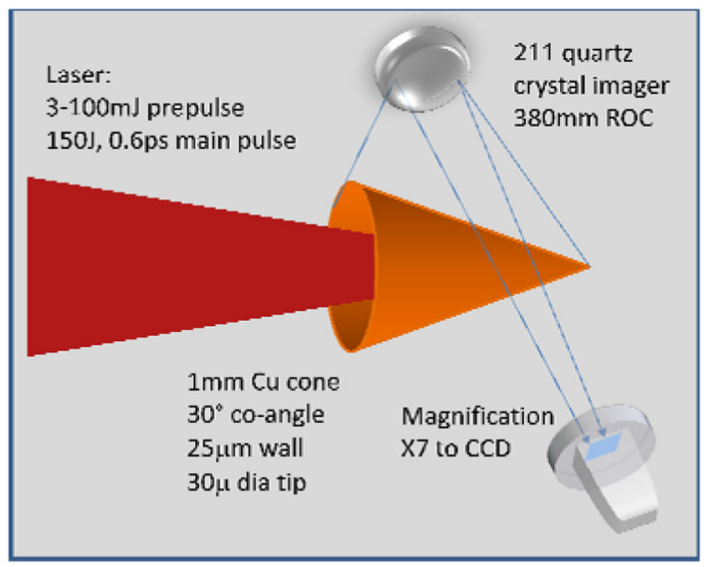

FIG. 1 (color online). Target detail illustrating $K_{\alpha}$ imaging geometry. of the hot electrons; hence the summation can be performed over the electron distribution alone, neglecting losses due to $K_{\alpha}$ production and significantly simplifying the calculation.

The $\mathrm{Cu} K_{\alpha}$ images are shown in Fig. 2(a). The lower and upper parts of the figure are data from $\mathrm{Cu}$ cone targets irradiated using the Titan laser described above, with 7.5 and $100 \mathrm{~mJ}$ prepulse, respectively. There are clear and striking differences between the low and high prepulse case as illustrated by the on-axis lineouts superimposed on the images.

For the $7.5 \mathrm{~mJ}$ low prepulse the $K_{\alpha}$ emission rises sharply, with a well-defined peak $50 \mu \mathrm{m}$ from the tip, before decreasing rapidly over the next $200 \mu \mathrm{m}$. For the $100 \mathrm{~mJ}$ high prepulse case the distribution is much broader peaking $200 \mu \mathrm{m}$ from the cone tip and extending a further $500 \mu \mathrm{m}$. These data are completely consistent with preplasma extending further into the cone, forcing the hot electron source further from the cone tip.
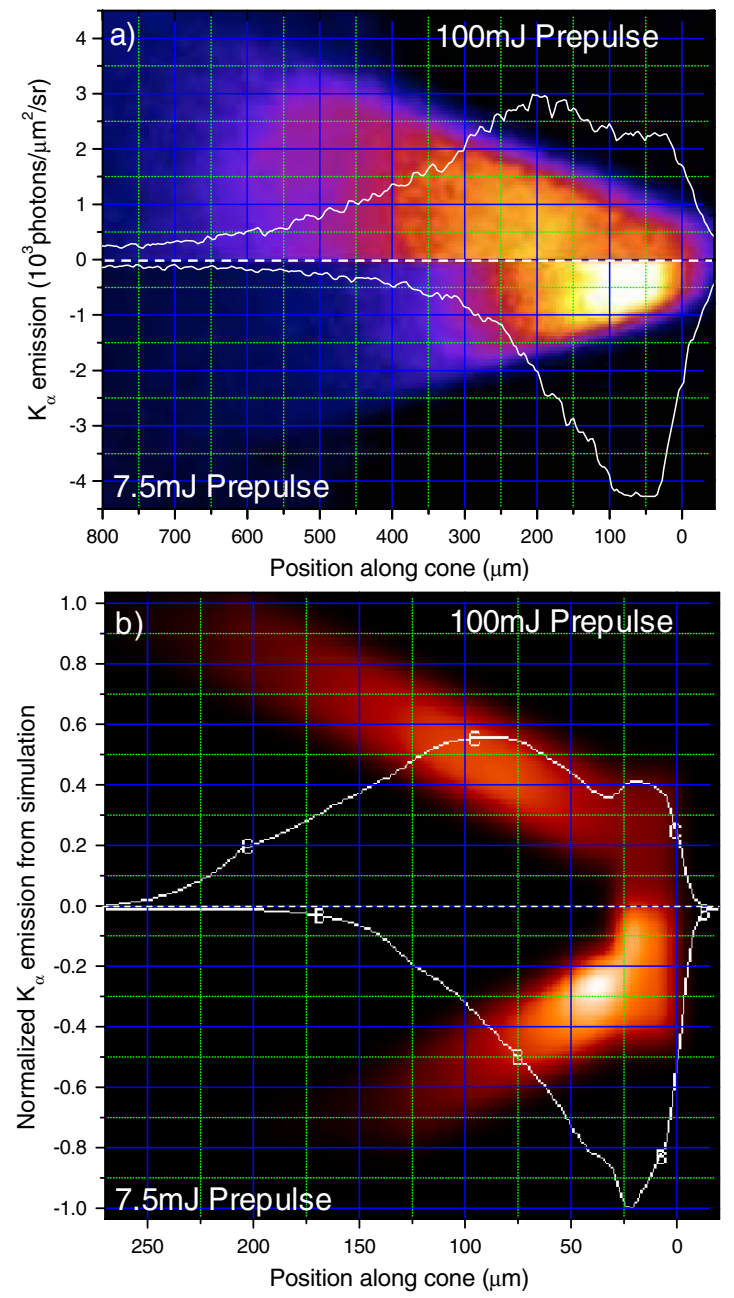

FIG. 2 (color online). (a) $K_{\alpha}$ images recorded for $100 \mathrm{~mJ}$ (upper) and $7.5 \mathrm{~mJ}$ (lower) prepulse. (b) Simulated $K_{\alpha}$ crosssectional images for the same shot parameters. Lineouts are onaxis $K_{\alpha}$ emission with line of sight opacity corrected for in (b). 
Direct comparison with the PSC/HYDRA modeling allows more insight into this process. The electron distributions from the PIC simulations were postprocessed to compute the time integrated $K_{\alpha}$ emission over the 3 ps. This was simulated by integrating over the electron energy distribution function and the electron cross section for $K_{\alpha}$ production. Figure 2(b) shows the resulting $K_{\alpha}$ deposition zones where $\iint n_{e}(E) \sigma_{K \alpha}(E) n_{\text {ion }} d v d t$ was calculated from particle dumps every $70 \mathrm{fs}$. The simulations reproduce the essential points from the experimental data, with a well-defined peak 30-50 $\mu \mathrm{m}$ from the cone tip for the low prepulse case and a broad distribution centered at $150 \mu \mathrm{m}$ for the $100 \mathrm{~mJ}$ prepulse.

The origin of these features is clear from examination of the laser interaction with the preformed plasma using the PSC PIC simulations as shown in Fig. 3. Here the laser Poynting flux is plotted at the time of peak main pulse fluence for both cases up to $4 \times 10^{20} \mathrm{~W} \mathrm{~cm}^{-2}$ using a red color ramp (left third of color bar). The preformed plasma used as the starting conditions for the PIC simulation is illustrated using a blue-green ramp (middle third) representing electron density up to $100 n_{c}$. The hot electron energy density above $1 \mathrm{MeV}$ normalized to 1 at the end of the $3 \mathrm{ps}$ simulation is illustrated using the green-white color ramp (right third). In the $7.5 \mathrm{~mJ}$ case the relativistic critical density surface on axis is $10 \mu \mathrm{m}$ from the inside tip of the cone. Rapid filamentation and self-focusing compared to the geometric focus (red dash) become apparent in the preformed plasma $\sim 60 \mu \mathrm{m}$ from the tip. In the $100 \mathrm{~mJ}$ prepulse case the relativistic critical density surface on axis is promoted an additional $30 \mu \mathrm{m}$ from the tip and selffocusing switches on at $\sim 120 \mu \mathrm{m}$. Best focus has an envelope approximately twice the initial diameter and has shifted an additional $\sim 30 \mu \mathrm{m}$ upstream.

Figure 3 illustrates that these simulations are in good qualitative agreement with the experiment. Multiple effects explain the quantitative differences: first, we did not account for transport effects over tens of picoseconds, where multi-MeV electrons will spray $K_{\alpha}$ emission over their mean-free path which is $\sim 1 \mathrm{~mm}$ in $\mathrm{Cu}$. This will likely extend the scale length of the $K_{\alpha}$ emission away from the tip of the cone. This would not significantly change the relative contrast between the cone tip and the wall, as the $K_{\alpha}$ bright spots in Fig. 2 are dominated by 100 $300 \mathrm{keV}$ electrons that have $<100 \mu \mathrm{m}$ mean-free-path. Second, we expect self-focusing in 3D to be stronger than that observed in the 2D simulation, which would promote the filamentation zone further from the cone tip, most significantly for the $100 \mathrm{~mJ}$ case. Both of these effects will tend to promote the simulated $K_{\alpha}$ emission zone towards that observed experimentally, but otherwise they will have no impact on the qualitative conclusions drawn.

The scale, duration, and resolution of the PIC simulation, along with the inclusion of beam filamentation and the radiative-hydro simulation of the initial preformed
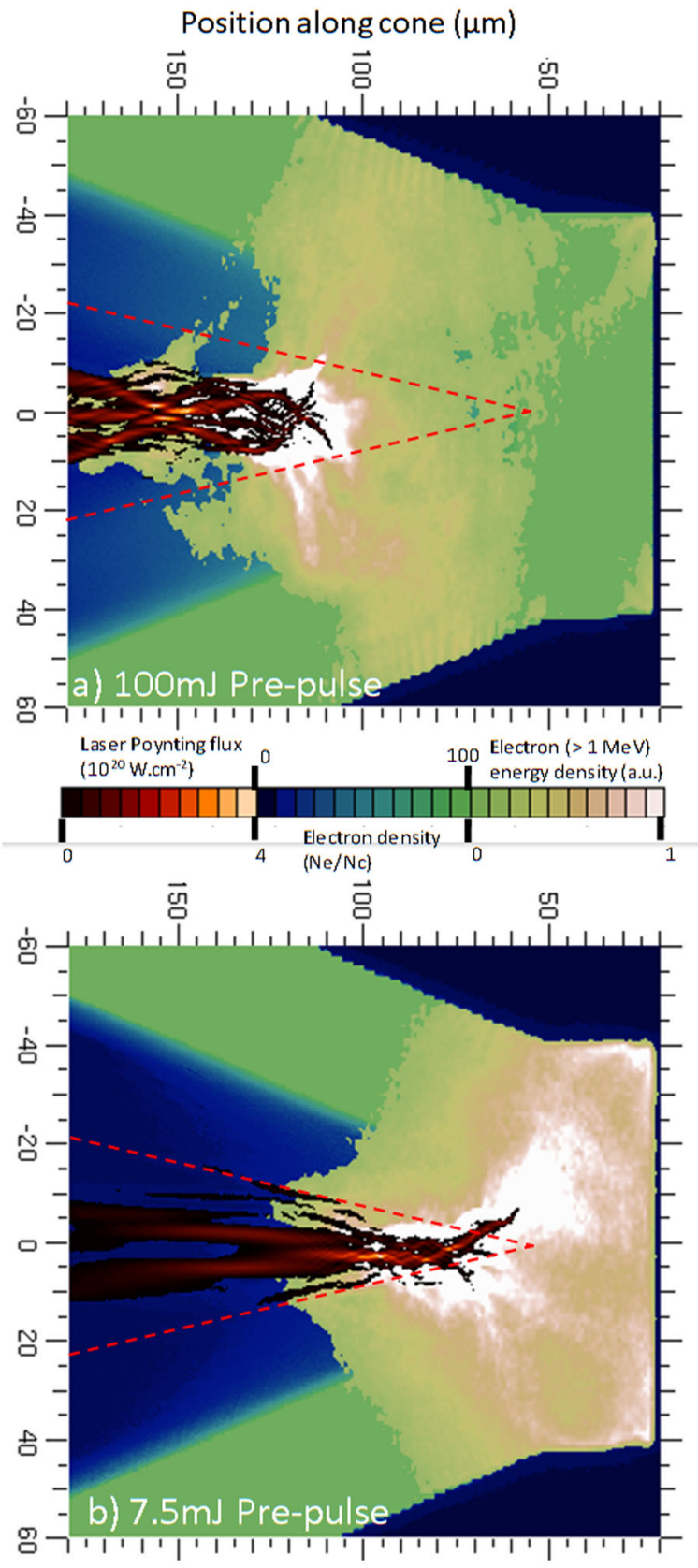

FIG. 3 (color online). Overlay of laser Poynting flux and electron density maps 1 ps after nominal peak fluence on target: (a) $100 \mathrm{~mJ}$ prepulse, (b) $7.5 \mathrm{~mJ}$ prepulse.

plasma is a significant step towards a fully integrated model of laser plasma interactions for fast-ignition cone targets. For an $f / 3,1 \mu \mathrm{m}$ laser beam at peak intensity $\sim 2 \times 10^{20} \mathrm{~W} \mathrm{~cm}^{-2}$, with $7.5 \mathrm{~mJ}$ prepulse, one main filament was observed to bore a hole through the short preplasma and reach the tip of the cone. The dynamics of relativistic filamentation lead to a very different picture depending on the preplasma scale length; for the $100 \mathrm{~mJ}$ prepulse case, all of the laser energy was diverted from the cone tip by the preformed plasma. The laser beam was split 
into multiple filaments far from best focus (and the tip of the cone), its propagation halted and energetic electrons generated mostly transverse to the incoming laser beam. Under these conditions a $100 \mathrm{~mJ}$ prepulse is clearly too great for effective coupling of hot electrons to the core of a fast-ignition fuel capsule. Limiting the prepulse is therefore critical for full scale FI and experiments at higher energy with longer pulse length and modeling with full scale injected pulses of $\sim 100 \mathrm{~kJ}$ in $\sim 20 \mathrm{ps}$ are required to see how the present work will extrapolate to ignition conditions.

This work performed under the auspices of the U.S. Department of Energy by Lawrence Livermore National Laboratory under Contract No. DE-AC52-07NA27344.

*macphee2@1lnl.gov

[1] M. Tabak et al., Phys. Plasmas 1, 1626 (1994).

[2] K. B. Wharton et al., Phys. Rev. E 64, 025401 (2001).

[3] F. Tavella et al., New J. Phys. 8, 219 (2006).

[4] L. Van Woerkom et al., Phys. Plasmas 15, 056304 (2008).

[5] S. D. Baton et al., Phys. Plasmas 15, 042706 (2008).
[6] N. Kumar et al., Phys. Scr. 73, 659 (2006), and references therein.

[7] M. H. Key et al., Phys. Plasmas 5, 1966 (1998); A. L. Lei et al., Phys. Rev. Lett. 96, 255006 (2006).

[8] C. D. Chen et al., Phys. Plasmas 16, 082705 (2009).

[9] M. Roth et al., Laser Part. Beams 23, 95 (2005).

[10] J. J. Honrubia et al., Nucl. Fusion 46, L25 (2006); C. Deutsch et al., Phys. Rev. Lett. 77, 2483 (1996).

[11] S. Atzeni, Phys. Plasmas 14, 052702 (2007).

[12] A Cotel et al., Appl. Phys. B 83, 7 (2006); A. Jullian et al., Opt. Lett. 30, 920 (2005).

[13] A. G. MacPhee et al., Rev. Sci. Instrum. 79, $10 \mathrm{~F} 302$ (2008).

[14] M. Marinak et al., Phys. Plasmas 5, 1125 (1998).

[15] H. Ruhl et al., in Introduction to Computational Methods in Many Particle Body Physics (Rinton, Paramus, NJ, 2006); M. Bonitz et al., Introduction to Computational Methods in Many Body Physics (Cambridge University Press, Cambridge, U.K., 2004); H. Ruhl, Collective SuperIntense Laser-Plasma Interaction (Habilitationsschrift, Technische Universität, Darmstadt, 2000).

[16] J. A. Koch, Rev. Sci. Instrum. 74, 2130 (2003).

[17] R. B. Stephens et al., Phys. Rev. E 69, 066414 (2004).

[18] K. U. Akli et al., Phys. Plasmas 14, 023102 (2007).

[19] Ch. Reich et al., Phys. Rev. Lett. 84, 4846 (2000). 